\title{
Outburst in OH17.7-2.0
}

\author{
M. Szymczak ${ }^{1}$, E. Gérard ${ }^{2}$ and P. Wolak ${ }^{1}$ \\ ${ }^{1}$ Toruń Centre for Astronomy, Nicolaus Copernicus University, Poland \\ email: msz@astro.uni.torun.pl \\ ${ }^{2}$ GEPI, Observatoire de Paris, France \\ email: Eric.Gerard@obspm.fr
}

\begin{abstract}
We present $\mathrm{OH} 1612 \mathrm{MHz}$ maser observations towards the pre-planetary nebula OH17.7-2.0 taken with the Nançay radio telescope in $2002-2007$. A bursting feature at $73 \mathrm{~km} \mathrm{~s}^{-1}$ grew by a factor of 11 in 740 days in left-hand circular polarization intensity, reaching a maximum in mid-2005. The $\mathrm{OH}$ flux density of features at neighbouring velocities exhibited a rise by a factor of 5-7. During the burst, the degree of circular polarization increased from 7 to $83 \%$. The blue-shifted emission showed weak variations of $7-10 \%$ in amplitude with the exception of the bluest feature for which the $\mathrm{OH}$ flux decreased with the rate of $\sim 4.4 \mathrm{Jy}_{\text {year }}{ }^{-1}$. Very unusual $\mathrm{OH}$ outbursts of individual features and remarkable changes in the shape of the maser profile appear to be linked with recent evolutionary changes in the detached shell.
\end{abstract}

Keywords. masers, stars: AGB and post-AGB, stars: individual: OH17.7-2.0, radio lines: stars

In 2003 the $\mathrm{OH} 1612 \mathrm{MHz}$ maser line profile of $\mathrm{OH} 17.7-2.0$ underwent a very unusual change; the intensity of the red-shifted emission at velocity $\sim 73 \mathrm{~km} \mathrm{~s}^{-1}$ increased nearly threefold over a period of $\sim 430$ days (Szymczak \& Gérard 2005). The follow-up VLBI observations revealed a bipolar morphology of the $1612 \mathrm{MHz}$ maser envelope with the bursting polarized emission coming from two unresolved components of brightness temperature up to $10^{11} \mathrm{~K}$ located at the edge of the biconical region $2500 \mathrm{AU}$ from the central star (Szymczak, Bartkiewicz \& Gérard 2006). Optical and NIR images confirm a bipolar morphology with two lobes separated by a dark waist (Sánchez Contreras et al. 2007). These observational facts bring further arguments that OH17.7-2.0 is in an intermediate evolutionary stage from asymptotic giant branch to planetary nebula.

After the detection of an outburst of the $\sim 73 \mathrm{~km} \mathrm{~s}^{-1}$ feature at $1612 \mathrm{MHz}$, we continued to monitor the target in a more systematic manner (typically twice a month) with the Nançay radio telescope. The 1612,1665 and $1667 \mathrm{MHz}$ lines were observed in full polarization mode. Here, we report the $1612 \mathrm{MHz}$ data taken with a velocity resolution of $0.14 \mathrm{~km} \mathrm{~s}^{-1}$ over a period of 5 years. The calibration uncertainty of the absolute flux density scale was $4-5 \%$.

Figure 1 shows the behaviour of 8 selected maser features in left- (LHC) and right-hand circular (RHC) polarization. Spectacular changes in $\mathrm{OH}$ flux appeared in the red-shifted $\left(\mathrm{V}>61.6 \mathrm{~km} \mathrm{~s}^{-1}\right)$ emission. A slow rise of the $73.0 \mathrm{~km} \mathrm{~s}^{-1}$ feature at LHC was seen; the polarized emission increased by a factor of 11 in 740 days. The maximum occurred near mid-2005 followed by a gradual decline since. The degree of circular polarization increased up to $83 \%$. A similar behaviour was observed for the neighbouring feature at $72.8 \mathrm{~km} \mathrm{~s}^{-1}$; the LHC flux density increased by a factor of about 5 . The maxima of both features coincide very well. The variations of the other neighbouring feature at $73.2 \mathrm{~km} \mathrm{~s}^{-1}$ differ considerably; the LHC flux density increased by a factor of 7 in 420 days followed by a high level plateau seen over about one year. A decline of this feature began 125 days prior to the maximum of the strongest feature. The RHC flux density variations of these three flaring features generally followed those in LHC but their amplitudes were 

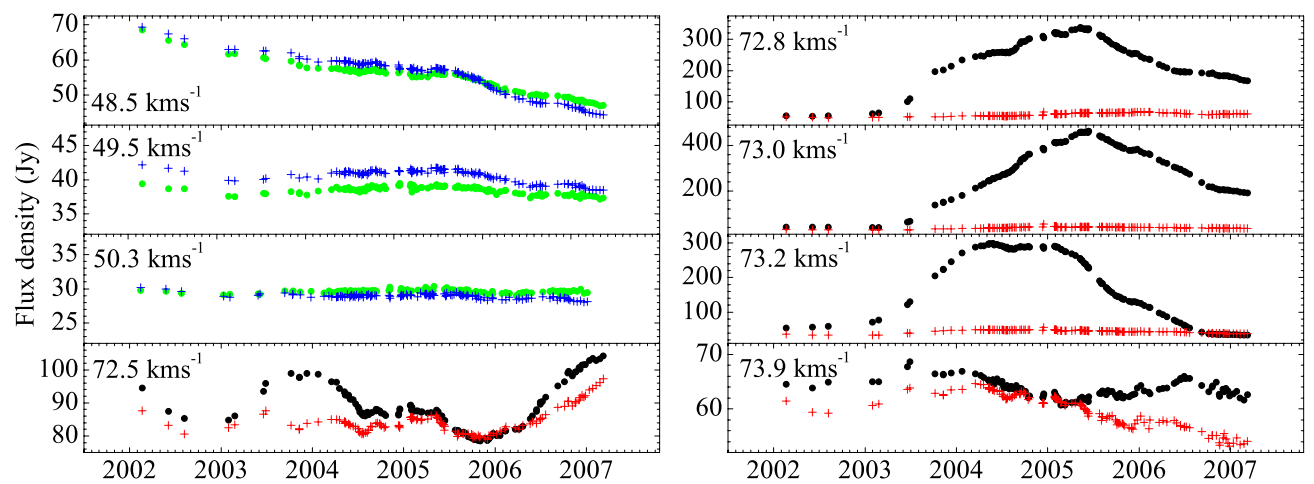

Figure 1. Flux density variations of the selected features in the $\mathrm{OH} 1612 \mathrm{MHz}$ maser spectrum of OH17.7-2.0. Each panel is labelled with the velocity of maser feature. The dots and crosses mark the left- and right-hand circular polarizations, respectively. Note in particular the burst of polarized emission at velocity $\sim 73 \mathrm{~km} \mathrm{~s}^{-1}$.

typically lower than $30 \%$. The other red-shifted features showed small amplitude $(\sim 25 \%)$ variations and their degrees of circular polarization were typically lower than $12 \%$.

The blue-shifted $\left(\mathrm{V}<61.6 \mathrm{~km} \mathrm{~s}^{-1}\right)$ emission did not exhibit dramatic changes. It is usually weakly polarized; the degree of circular polarization was always lower than $8 \%$. Features at 50.3 and $49.5 \mathrm{~km} \mathrm{~s}^{-1}$ showed $\mathrm{OH}$ amplitude variations of 7 and $12 \%$ respectively. However, the bluest feature of $48.5 \mathrm{~km} \mathrm{~s}^{-1}$ experienced a decline with a rate of about 4.4 $\mathrm{Jy}_{\text {year }}{ }^{-1}$ (Fig. 1).

We noted small amplitude variations in the $\mathrm{OH}$ flux of the blue-shifted emission (for instance the $49.5 \mathrm{~km} \mathrm{~s}^{-1}$ feature) and the RHC flux of the red-shifted emission. It is intriguing that the onset of the outburst coincided with a minimum of the $\mathrm{OH}$ flux while a maximum slightly preceded the outburst maximum. This suggests that there is a quiet $\mathrm{OH}$ emission emission that still exhibits regular variations with a period $\geqslant 1200$ days.

Our monitoring of OH17.7-2.0 documented well the behaviour of the $\mathrm{OH} 1612 \mathrm{MHz}$ maser emission over a period of 5 years. The most striking result, not seen from the data reported in Szymczak \& Gérard (2005), is a gradual decline of the bluest maser feature and evidence for periodic changes of several blue-shifted features as well as the RHC flux of red-shifted features. This seems to be consistent with a scenario in which the outburst emission arises in a very confined region of interaction of the jet-like outflow from the central star with the detached OH shell (Szymczak, Bartkiewicz \& Gérard 2006), while the $\mathrm{OH}$ maser from the overall AGB shell still varies in a periodic manner as observed in $\mathrm{OH} / \mathrm{IR}$ objects (Herman \& Habing 1985). The $\mathrm{OH}$ maser emission from OH17.7-2.0 appears to be a good probe of recent evolutionary changes in the detached shell, which are presumably induced by bipolar winds. The present case study of $\mathrm{OH} 17.7-$ 2.0 demonstrates the need for both long-term monitoring of well selected $\mathrm{OH}$ sources and prompt follow-up VLBI observations of outstanding events if one wants to understand the complex physics of circumstellar $\mathrm{OH}$ masers.

\section{References}

Herman, J. \& Habing, H. J. 1985, A\&AS 59, 523

Sánchez Contreras, C., Le Mignant, D., Sahai, et al. 2007, ApJ 656, 1150

Szymczak, M., Bartkiewicz, A. \& Gérard, E. 2006, Proc. 8th EVN Symposium (submitted)

Szymczak, M. \& Gérard, E. 2005, A\&\&A 433, L29 